\title{
Mapeo sistemático de la literatura de políticas docentes de educación básica de la Reforma Educativa
}

\section{A systematic mapping of the literature on the basic education teaching policies of the Educational Reform}

DOI: https://doi.org/10.32870/dse.v0i24.1038

Dolores Graciela Cordero Arroyo*

María del Ángel Vázquez Cruz**

Claudia Navarro Corona***

\section{Resumen}

En el sexenio 2012-2018, México vivió una Reforma Educativa que afectó la normatividad y estructura de la carrera docente. Este estudio es un mapeo sistemático de la producción de la investigación educativa acerca de las políticas para docentes de educación básica en servicio publicadas de enero 2013 a junio de 2020. Se estudiaron tres dimensiones de políticas docentes: carrera docente, formación continua e instituciones y procesos de las políticas de profesores. Se encontró un total de 97 documentos, 58 centrados en la carrera docente, 24 referentes a la formación continua y 15 estudios de instituciones y procesos de las políticas docentes. Se concluye que la investigación educativa priorizó el estudio de las políticas referidas a la evaluación de la carrera docente y las políticas de formación continua quedaron en segundo término. El mapeo proporciona información sistematizada para que el lector trace rutas propias de exploración para comprender las lecciones de nuestro pasado reciente en esta temática.

Palabras clave: políticas docentes - evaluación docente - formación continua - revisión de la literatura reforma educativa.

\section{Abstract}

In the 2012-2018 presidential term Mexico underwent an Educational Reform that affected the regulations and structure of teacher training. This study is a systematic mapping of the educational research production on policies for in-service basic education teachers published from January 2013 to June 2020. Three dimensions of teaching policies were studied: teacher training, ongoing training, and institutions and processes of teacher policies. We found 97 documents, 58 of which focused on teacher training, 24

* Doctora en Filosofía y Ciencias de la Educación. SNI 2. Línea de investigación: formación de profesores. Investigadora Titular del Instituto de Investigación y Desarrollo Educativo de la Universidad Autónoma de Baja California. México. gcordero@uabc.edu.mx

** Maestra en Ciencias Educativas. Línea de investigación: formación de profesores. Docente en la Facultad de Ciencias Administrativas y Sociales de la Universidad Autónoma de Baja California. México.vazquezm87@uabc.edu.mx

*** Doctora en Ciencias Educativas. SNI 1. Línea de investigación: Liderazgo y gestión de instituciones educativas, formación de docentes y directivos. Profesora-investigadora en la Escuela de Humanidades y Educación del Tecnológico de Monterrey. México.

c.navarrocorona@gmail.com 
on ongoing training and 15 studies of institutions and processes of teaching policies. We concluded that educational research prioritized the study of policies related to the evaluation of teacher training, followed by ongoing training policies. This mapping provides systematized information for the reader to trace their own exploration routes to understand the lessons of our recent past in this area.

Keywords: teacher policies - teacher evaluation - teacher professional development - Literature review - educational reform.

\section{Introducción}

Con el fin de mejorar la calidad de la educación, organismos internacionales como la Oficina Regional de Educación para América Latina y el Caribe de Organización de las Naciones Unidas para la Ciencia y Cultura (OREALC-UNESCO), la Organización para la Cooperación y el Desarrollo Económicos (OCDE) y la Organización de Estados Iberoamericanos para la Educación, la Ciencia y la Cultura (OEI) han desarrollado estudios y recomendaciones en distintos ámbitos de la educación.

En las últimas décadas, el profesorado de educación básica ha sido uno de los temas más atendidos tanto en los países europeos como en los latinoamericanos (Ducoing, 2019). Estos organismos han realizado reuniones de trabajo y publicado estudios, orientaciones y directrices para el diseño de políticas docentes, con fines de fortalecer su perfil profesional y hacer evidente sus condiciones de trabajo.

Así, en este siglo, las políticas docentes, entendidas como aquellas "acciones que forman parte de un plan o proyecto de Estado que se dirige a los maestros de educación básica" (Cuevas, Inclán, 2021: 355), son discutidas por una multiplicidad de organismos internacionales (Bruns, Luque, 2015; Elacqua et al., 2018), o de la sociedad civil (Mexicanos Primero, 2016) y se han convertido en un componente fundamental de cualquier política educativa.

La Oficina Regional de Educación para América Latina y el Caribe de la Organización de las Naciones Unidas para la Educación, la Ciencia y la Cultura (OREALC-UNESCO, 2013), distingue tres dimensiones de las políticas docentes: $a$ ) la formación inicial, $b$ ) la formación continua y c) la carrera docente.

Las dos primeras dimensiones corresponden a las fases principales de la formación del profesorado (Ávalos, 2007). La formación inicial y la formación en el servicio tienen características propias que las diferencian. La formación inicial es un periodo formativo acotado a cuatro años de licenciatura, se imparte en instituciones especializadas de educación superior (genéricamente denominadas escuelas normales) con planes de estudios definidos y regulados por la federación en el ámbito nacional. La formación continua o en el servicio, por su parte, tiene características distintas. Esta formación se ofrece a los actores educativos a lo largo de su vida profesional, desde el ingreso hasta la jubilación. Puede tener diversos fines específicos, como 
la actualización, la nivelación o la capacitación. Es ofrecida por instancias formadoras públicas o privadas en acciones genéricamente (y de manera errónea) denominadas como capacitación (Birgin, 2012; Cordero et al, 2017).

Un tercer elemento presente en toda política docente es la carrera docente; ésta se entiende como el "sistema de ingreso, ejercicio, estabilidad, desarrollo, ascenso y retiro de las personas que ejercen la profesión docente" (Murillo, 2007: 37). Entre los estudios internacionales, destaca el trabajo de Cuenca (2015) acerca de los lineamientos de las carreras docentes en 18 países de América Latina.

En México, en el sexenio 2012-2018 hubo un importante cambio en la regulación de las políticas docentes, denominado Reforma Educativa. La reforma de los artículos $3^{\circ}$ y 73 de la Constitución Política de los Estados Unidos Mexicanos (DOF, 2013a), la Ley General de Educación (DOF, 2013b), y la promulgación de la Ley General del Servicio Profesional Docente (LGSPD) (DOF, 2013c) y de la Ley del Instituto Nacional para la Evaluación de la Educación (DOF, 2013d) concretaron un nuevo esquema de carrera docente.

Este periodo ha sido ampliamente reseñado y analizado por Ornelas (2018). Este autor sostiene que antes de la reforma, en nuestro país la tecnología del poder que el Estado mexicano tenía vía el corporativismo sindical bastaba para controlar su figura, su función, su valor y su trabajo. En 2013 esta situación cambió. La reforma constitucional y legal pretendió devolver al Estado el control de la regulación de la carrera docente.

La LGSPD instituyó el Servicio Profesional Docente (SPD) como el conjunto de actividades y mecanismos para los procesos de ingreso, promoción, permanencia y reconocimiento del personal docente y personal con funciones directivas en el servicio con el fin de garantizar su idoneidad (DOF, 2013c). El SPD definió la evaluación del desempeño docente como el mecanismo para el tránsito por estos procesos, conformando una nueva estructura de carrera que ha sido calificada como meritocrática (Cuenca, 2015; Cuevas, Rangel, 2019). En el sexenio, los esfuerzos de las autoridades educativas federales y estatales se centraron en el desarrollo e implementación de las políticas relacionadas con los procesos de evaluación para el ingreso, la promoción y la permanencia en la función (Cordero et al., 2017).

Si bien en sexenios anteriores se habían implementado programas para evaluar a los docentes de educación básica, la evaluación obligatoria del desempeño con fines de permanencia fue una condición inédita en el país (Cuevas, Rangel, 2019). De ahí que la "permanencia" en el servicio fue un tema central en el discurso de fuerzas políticas que se oponían a las nuevas disposiciones sobre la carrera docentes implementadas en el sexenio, pues afirmaban que era una reforma política, no educativa, y que las consecuencias de dicha evaluación afectaban los derechos laborales de los trabajadores (Cuevas, Rangel, 2019; Paoli, 2013; Rodríguez, 2016).

Las políticas docentes surgieron como un tema de polémica social y la Reforma Educativa generó mucho interés entre investigadores educativos, por lo que se publicó ampliamente 
sobre el tema. Los interesados en comprender esta etapa de las políticas docentes en nuestro país encuentran una producción dispersa publicada en diversos medios. Dada esta situación, se consideró de interés realizar un mapeo sistemático de la producción académica publicada de enero de 2013 a junio de 2020, que toma como objeto de estudio las políticas docentes de educación básica asociadas a la Reforma Educativa de 2013 implementada en México.

La investigación, como fundamento de la práctica y la política educativa, requiere de una incrementada capacidad para recuperar conocimiento, interpretarlo y tomar decisiones derivadas de este proceso. La producción académica aporta beneficios directos o indirectos a la educación, aunque el acceso que los distintos actores tienen al conocimiento académico especializado mantiene cierto grado de complejidad (Wagner et al., 1999). Por esta razón, aunque la Reforma Educativa fue derogada el 15 de mayo de 2019, el contar con un mapeo sistemático resulta relevante pues organiza la producción académica, la hace accesible y permite que los investigadores y tomadores de decisiones puedan construir o analizar las políticas vigentes al "tomar en consideración las lecciones del pasado" (Del Castillo, 2019: 2).

\section{Procedimiento metodológico}

El mapeo sistemático de literatura (MSL) y la revisión sistemática de literatura (RSL) son estudios de tipo secundario (Keele, 2007). Ambos se centran en el análisis de investigaciones primarias, sin embargo, no deben confundirse entre sí, pues cada uno atiende a propósitos diferenciados.

Celdrán-Bernabeu et al. (2018) identifican notorias diferencias entre ambas metodologías. El MSL explora un tópico o tema, identifica, selecciona y organiza los estudios primarios. Su propósito es la identificación de tendencias de comportamiento métrico y clasificatorio de los estudios en un campo del saber.

El MSL se define mediante el establecimiento de un protocolo dado con la especificación de preguntas exploratorias que rigen la estrategia de búsqueda, la integración de metadatos y la extracción de resultados. Un MSL permite trazar un dominio con alto nivel de gradualidad para reconocer grupos de evidencias y desiertos de evidencias de cara al desarrollo de nuevas revisiones y estudios (Celaya et al., 2020). En este sentido, la contribución de los mapeos a la literatura es la organización de la producción y no propiamente las abstracciones teóricas o su discusión. Así, un MSL puede ser un estudio preparatorio o introductorio a una RSL. La RSL, por su parte, trata de dar respuesta a preguntas de investigación y, en función de esto, discrimina estudios respecto a su impacto o relevancia a fin de explicar y discutir las tendencias del conjunto.

Petersen et al. (2008) constituyen uno de los referentes más recurridos en los mapeos sistemáticos. Estos autores definen el proceso en cuatro pasos con objetivos precisos: 1) definición de las preguntas de investigación para establecer el alcance de la investigación, 2) conducción de la búsqueda para identificar la producción, 3) visualización de la producción para seleccionar los trabajos, 4) identificación de palabras clave usando resúmenes para clasificar 
temáticamente y 5) extracción de datos para obtener resultados. Estos pasos han sido tomados como referentes en otros estudios como el de Monroy et al. (2016) para explorar el tema de arquitectura de software; el de Villar et al. (2013) para estudiar el concepto de tendencia técnica, y el de Navarro-Corona y Ramírez-Montoya (2017) para mapear la producción sobre evaluación docente.

Para el desarrollo de este estudio se tomó como referencia la propuesta diseñada por Navarro-Corona y Ramírez-Montoya (2018), a partir de los planteamientos de Petersen et al. (2008) y Sinoara et al. (2017). El proceso que proponen las autoras consta de cuatro fases: a) planteamiento, b) localización de la producción académica, c) preanálisis, y d) análisis. Los pasos de cada fase se incluyen en la tabla 1.

Tabla 1. Fases y pasos del mapeo sistemático

\begin{tabular}{ll}
\hline \multicolumn{1}{c}{ Fases } & \multicolumn{1}{c}{ Pasos } \\
\hline Planteamiento & Definición del objeto de estudio. \\
& Diseño de las preguntas de investigación. \\
& Selección de las fuentes de búsqueda. \\
\hline Localización de la producción & Refinamiento de los criterios de búsqueda. \\
académica & Búsqueda de la producción académica. \\
\hline Preanálisis & Acercamiento al contenido de la producción académica. \\
& Diseño de base de datos. \\
\hline Análisis & Registro de los documentos en las bases de datos \\
& Análisis de las tendencias. \\
\hline
\end{tabular}

Fuente: Elaboración propia a partir de Navarro y Ramírez (2018).

\section{Fase A. Planteamiento}

En la fase de planteamiento se definió el objeto de estudio, se diseñaron las preguntas de investigación y se establecieron las fuentes de búsqueda de los documentos.

1. Definición del objeto de estudio. Esta investigación tuvo como objeto de estudio la producción académica con respecto a las políticas docentes en educación básica asociadas a la Reforma Educativa de 2013 y publicadas de enero 2013 a junio de 2020. Se recuperó la propuesta OREALC-UNESCO (2013), que distingue tres dimensiones de las políticas docentes: a) la formación inicial, b) la formación continua y c) la carrera docente. No obstante, para fines de esta investigación, se retomaron dos de estas dimensiones: carrera docente y formación continua. Se excluyó la dimensión formación inicial dado que el interés de este estudio está centrado en la figura del profesorado en servicio. Además, la formación continua es un objeto de estudio menos atendido por la investigación educativa. Tal como plantea Vezub (2021: 31), este campo "ha sido un campo fértil en prácticas y experiencias, más profuso en políticas, intervenciones y programas, pero ha sido poco abordado desde la investigación educativa". 
Adicionalmente, se propuso e incluyó una dimensión que hace referencia al estudio de las instituciones y procesos de las políticas docentes en general. En la tabla 2 se presenta la definición de las tres dimensiones que estudian en este mapeo sistemático.

Tabla 2. Definición del concepto políticas docentes de acuerdo con la UNESCO (2013)

\begin{tabular}{|l|l|}
\hline \multicolumn{1}{|c|}{ Dimensión } & \multicolumn{1}{c|}{ Definición } \\
\hline Formación continua & $\begin{array}{l}\text { Procesos de mejora profesional continua, que inician con los } \\
\text { programas de inducción a la docencia y atraviesan la carrera } \\
\text { docente hasta el final de la misma. }\end{array}$ \\
\hline Carrera docente & $\begin{array}{l}\text { Regulaciones para el ejercicio de la docencia que determinan } \\
\text { el ingreso, promoción, reconocimiento y permanencia en la } \\
\text { profesión. }\end{array}$ \\
\hline $\begin{array}{l}\text { Instituciones y procesos de las polí- } \\
\text { ticas docentes }\end{array}$ & $\begin{array}{l}\text { Procesos y factores que afectan la formulación e implementa- } \\
\text { ción de las políticas docentes. }\end{array}$ \\
\hline
\end{tabular}

Fuente: Elaborada a partir de OREALC-UNESCO (2013).

Así, se revisó la producción académica de las tres dimensiones que corresponden a las políticas de docentes en servicio: 1) formación continua, 2) carrera docente y 3) instituciones y procesos de dichas políticas.

2. Diseño las preguntas de investigación. En la tabla 3 se exponen las preguntas y categorías de la investigación, que tuvieron como propósito específico caracterizar la producción académica respecto a las políticas docentes en las tres dimensiones antes mencionadas.

Tabla 3. Preguntas de investigación de este estudio

\begin{tabular}{|c|c|c|}
\hline Pregunta & Categoría & Subcategoría \\
\hline $\begin{array}{l}\text { ¿Qué formato de publicación } \\
\text { tienen los textos académicos } \\
\text { identificados? }\end{array}$ & $\begin{array}{l}\text { Tipo de docu- } \\
\text { mento. }\end{array}$ & $\begin{array}{l}\text { - Ponencia, artículo, estudio INEE, capítu- } \\
\text { lo de libro. }\end{array}$ \\
\hline $\begin{array}{l}\text { ¿Qué tendencias de produc- } \\
\text { ción se observan en el perio- } \\
\text { do comprendido entre enero } \\
\text { de } 2013 \text { a junio de } 2020 \text { en el } \\
\text { tema? }\end{array}$ & $\begin{array}{l}\text { Tendencias de } \\
\text { producción por } \\
\text { año. }\end{array}$ & - Años de 2013 a 2020. \\
\hline $\begin{array}{l}\text { ¿Qué dimensiones de las po- } \\
\text { líticas docentes se abordan } \\
\text { en las investigaciones? }\end{array}$ & $\begin{array}{l}\text { Dimensión de } \\
\text { las políticas do- } \\
\text { centes }\end{array}$ & $\begin{array}{l}\text { - Carrera docente, formación continua, } \\
\text { instituciones y procesos de las políticas } \\
\text { docentes. }\end{array}$ \\
\hline \multirow[t]{2}{*}{$\begin{array}{l}\text { ¿A qué subdimensión de las } \\
\text { políticas docentes se refiere? }\end{array}$} & $\begin{array}{l}\text { Etapas de la carre- } \\
\text { ra docente }\end{array}$ & $\begin{array}{l}\text { - Ingreso e iniciación, promoción y reco- } \\
\text { nocimiento, permanencia. }\end{array}$ \\
\hline & $\begin{array}{l}\text { Ámbito de aná- } \\
\text { lisis }\end{array}$ & $\begin{array}{l}\text { - Instituciones, leyes y documentos oficia- } \\
\text { les, percepciones y reflexiones del profe- } \\
\text { sorado. }\end{array}$ \\
\hline
\end{tabular}


3. Selección de las fuentes de búsqueda. La búsqueda de las publicaciones se realizó en medios impresos y electrónicos. Se consideraron artículos en revistas académicas, ponencias, estudios publicados por el Instituto Nacional para la Evaluación de la Educación (INEE) y capítulos de libros. Para la búsqueda de los artículos se seleccionaron tres bases de datos: 1) la Red lberoamericana de Innovación y Conocimiento Científico (Redib), 2) la Red de Revistas Científicas de América Latina y el Caribe (Redalyc), y 3) Ebsco Host. En esta búsqueda se excluyeron los textos editoriales, las reseñas de libros y entrevistas.

En cuanto a las ponencias, se revisaron los archivos de los Congresos Nacionales de Investigación Educativa de 2015, 2017 y 2019, organizados cada dos años por el Consejo Mexicano de Investigación Educativa (COMIE). Se consideró este congreso por ser el evento académico del ámbito educativo con mayor relevancia del país. Otro de los espacios en los que se realizó la búsqueda fue el repositorio de documentos del INEE, específicamente las publicaciones de la colección "Estudios e investigaciones" que abordan temas relacionados con las políticas docentes en educación básica.

\section{Fase B. Localización de la producción académica}

En esta fase se afinaron los criterios para la selección de la producción académica y se realizó la búsqueda de las ponencias, artículos, estudios del INEE y capítulos de libros.

4. Refinamiento de los criterios de búsqueda. Se precisaron las características de la producción académica. Para la localización de la producción se utilizó como concepto clave "Servicio Profesional Docente", por ser el nombre dado a la carrera docente en México durante el sexenio 2013-2018 (DOF, 2013c). Los textos en cada etapa se seleccionaron a partir de los siguientes criterios de inclusión:

- Palabra clave: Servicio Profesional Docente

- Periodo de publicación: enero de 2013 a junio de 2020.

- Tipo de trabajo: Estudio empírico, estudio documental y ensayo.

- Nivel educativo: Educación básica.

- Sector: Público.

- Dimensiones de las políticas docentes analizadas en la primera etapa: a) carrera docente, b) formación continua, y c) instituciones y procesos de las políticas docentes.

5. Búsqueda de la producción académica. Se recuperó y etiquetó la producción académica que cumplía con los criterios de investigación. A continuación, se explica el proceso y el número de estudios encontrados por tipo de documento.

Artículos. Al realizar la búsqueda de textos en las bases de datos se utilizaron los siguientes criterios de búsqueda: 
- Palabra clave: "Servicio Profesional Docente".

- Campo: Texto completo.

- Match: Coinciden todos los términos. ${ }^{1}$

- Tipo de recurso: Archivo de revista.

- Rango de publicación: 2013 a junio, 2020.

- País: México.

La Redib arrojó como resultado 15 textos, de los cuales seis cumplieron con los criterios de selección. En Redalyc se encontraron 64 textos y siete fueron pertinentes para este estudio. Ebsco Host dio como resultado 58 artículos y se seleccionaron 11.

Ponencias. Las memorias de los congresos del COMIE se dividieron por líneas de investigación. En este estudio sólo se revisaron las que se consideró tenían relación con el tema de políticas docentes. En los archivos del Congreso 2015 se revisaron las temáticas c) Políticas y gestión en la educación y e) Procesos de formación. En las memorias de 2017 y 2019 se analizaron las áreas temáticas 8) Procesos de formación, 9) Sujetos de la educación y 10) Política y gestión de la educación. En total se descargaron 40 textos.

Estudios del INEE. En el buscador del repositorio del INEE no es posible realizar búsquedas por tipo de publicación, por lo que para identificar los textos pertinentes se ingresó a la colección "Estudios e Investigaciones" y se realizó la lectura de los títulos de los documentos.

Capítulos de libros. Se localizaron ocho libros publicados en México con respecto a la Reforma Educativa de 2013, en los que se incluyeron capítulos de temas relacionados con las políticas docentes. Cinco de estas publicaciones forman parte de una colección diseñada por el INEE y el Fondo de Cultura Económica, y tres son libros electrónicos de acceso abierto de otras editoriales. En el ámbito internacional, se encontró un libro que documenta experiencias de formación continua de distintos países, entre ellos, México. Para fines del presente artículo, se consideraron como unidades de análisis solamente los capítulos que tenían como objeto de estudio los procesos del SPD en alguna de las tres dimensiones ya mencionadas.

\section{Fase C. Preanálisis}

Esta fase implicó realizar una primera lectura de los textos para corroborar su pertinencia y se diseñó la base de datos para registrar la producción académica.

6. Acercamiento al contenido de la producción académica. Se hizo la lectura de los textos para analizar su pertinencia.

1 Se refiere a que se presenten los textos en los que se encuentra el enunciado "Servicio Profesional Docente" de manera textual y se excluyen los textos en los que se menciona sólo uno de los conceptos. 
Artículos. Se identificaron en las bases de datos Rebid, Redalyc y Ebsco Host diez revistas con textos pertinentes para este estudio, de las cuales siete son nacionales: Revista de Investigación Educativa (CPU-e), Revista Mexicana de Análisis Político y Administración Pública (REMAP), Revista Electrónica de Investigación e Innovación Educativa (REIIE), Revista de Investigación Educativa de la Red de Investigadores Educativos de Chihuahua (IE-REDIECH), Revista Mexicana de Investigación Educativa (RMIE), Revista Latinoamericana de Estudios Educativos (RLEE), El Cotidiano - Revista de Ia Realidad Mexicana Actual; dos de Estados Unidos: Journal of Labor and Society (JLS) y Education Policy Analysis Archives (EPAA); y una española: Revista Iberoamericana de Evaluación Educativa (RIEE). En la tabla 4 se precisa el número de artículos encontrados en cada revista.

Tabla 4. Concentrado de artículos identificados por revista

\begin{tabular}{|l|l|l|l|}
\hline Base de datos & Revista & $\mathbf{N}$ & \% \\
\hline \multirow{4}{*}{ Rebid } & CPU-e & 1 & 5 \\
\cline { 2 - 4 } & REMAP & 1 & 5 \\
\cline { 2 - 4 } & REIIE & 2 & 10 \\
\cline { 2 - 4 } & RIEE & 2 & 10 \\
\hline \multirow{3}{*}{ Redalyc } & IE -REDIECH & 1 & 5 \\
\cline { 2 - 4 } & RMIE & 1 & 5 \\
\cline { 2 - 4 } & RLEE & 1 & 5 \\
\hline \multirow{3}{*}{ Ebsco Host } & EPAA & 7 & 35 \\
\cline { 2 - 4 } & El cotidiano & 3 & 15 \\
\cline { 2 - 4 } & JLS & 1 & 5 \\
\hline \multirow{2}{*}{ Total } & & 20 & 100 \\
\hline
\end{tabular}

Si bien sólo se localizaron 20 publicaciones en las bases de datos que se revisaron, es posible que hubiera un mayor número de textos que estudien las políticas docentes en revistas que no se encuentran indexadas a Redib, Redalyc o Ebsco Host.

Ponencias. La tabla 5 presenta un concentrado con el número y porcentaje de ponencias identificadas referentes a las políticas docentes, por año y línea de investigación presentadas en los congresos del COMIE. 2017 es el año que cuenta con más publicaciones. En 2019 el número de ponencias en esta temática se redujo casi a la mitad. 
Tabla 4. Concentrado del número de ponencias publicadas por el COMIE

\begin{tabular}{|l|l|l|l|}
\hline Año & Línea & N & $\%$ \\
\hline \multirow{3}{*}{2015} & Temática general C. Políticas y gestión en la educación & 4 & 10.0 \\
\cline { 2 - 5 } & Temática general E. Procesos de formación & 5 & 12.5 \\
\hline \multirow{3}{*}{2017} & Línea 8. Procesos de formación & 4 & 10.0 \\
\cline { 2 - 5 } & Línea 9. Sujetos de la educación & 6 & 15.0 \\
\cline { 2 - 5 } & Línea 10. Política y gestión de la educación & 10 & 25.0 \\
\hline \multirow{3}{*}{2019} & Línea 8. Procesos de formación & 5 & 12.5 \\
\cline { 2 - 5 } & Línea 9. Sujetos de la educación & 2 & 5.0 \\
\cline { 2 - 4 } & Línea 10. Política y gestión de la educación & 4 & 10.0 \\
\hline & Total & 38 & 100 \\
\hline
\end{tabular}

Estudios del INEE. De los 33 documentos de la colección "Estudios e Investigaciones", 22 tuvieron como objeto de análisis las políticas docentes.

Capítulos de libros. En los ocho libros recuperados que analizan la Reforma Educativa de 2013, se encontraron 17 capítulos que cumplieron con los criterios de inclusión. Es importante destacar que 16 fueron ensayos y sólo uno de investigación documental.

Se consideraron en total 97 documentos con respecto a las políticas docentes en México en el marco de la Reforma Educativa de 2013, clasificados de la siguiente manera: 38 ponencias, 20 artículos, 22 estudios del INEE y 17 capítulos de libro. Cada archivo se guardó en electrónico con una etiqueta compuesta por el tipo de documento: ponencias $[P]$, artículos [A], estudios INEE [E] y capítulos de libros [C], y el número de archivo. Esa misma clave se utilizó como variable de identificación en la base de datos.

7. Diseño de base de datos. Se elaboró un formulario de Google para registrar los textos identificados. Se incluyeron los siguientes campos: año de publicación, título, autor(es), tipo de documento, fuente y dimensión de estudio.

\section{Fase D. Análisis}

8. Registro de los documentos en la base de datos. Se realizó la captura de la información en el formulario correspondiente. En el caso de las ponencias y artículos se llevó a cabo una lectura del resumen de cada archivo y se llenó el formulario. Para los estudios del INEE y capítulos de libros se realizó la lectura del apartado introductorio. En algunos documentos fue necesario hacer una lectura del texto completo para poder recabar la información necesaria. 
9. Análisis de la tendencia respecto a la producción académica que toma como objeto de estudio las políticas docentes. A partir del formulario se generó una base de datos en Excel con los textos que contenían información respecto a las políticas docentes de educación básica en el sexenio 2012-2018. La base de dato en Excel se depuró e importó al programa SPSS, en el que se generaron las gráficas y tablas para realizar los análisis y presentar los resultados.

\section{Resultados}

A continuación, se describen las características de la producción académica que estudia las políticas docentes en el marco de la reforma educativa que se implementó en México en el año 2013. La lista de los documentos mapeados se encuentra en una página de internet, ${ }^{2}$ a fin de que el lector interesado en estos temas pueda consultar los textos de su interés. Para cada uno de ellos se integró una clave con la inicial del tipo de documento y su número de registro en la base de datos.

Tipo de documento. En la tabla 6 se presenta una comparación del número de documentos encontrados por tipo de publicación. Como se puede observar, la mayoría de los textos que incluye este estudio son ponencias del congreso organizado por el COMIE.

Tabla 5. Estudios de políticas docentes por tipo de publicación

\begin{tabular}{lll}
\hline Tipo de documento & Frecuencia & Porcentaje \\
\hline Ponencias & 38 & 39.2 \\
\hline Estudios INEE & 22 & 22.7 \\
\hline Artículos & 20 & 20.6 \\
\hline Capítulos de libros & 17 & 17.5 \\
\hline Total & 97 & 100.0 \\
\hline
\end{tabular}

Tendencias de producción por año. Como se observa en la tabla 7, se identificó que 58 (59.7\%) de los textos se refirieron a la carrera docente, es decir, se enfocaron a los procesos de evaluación del desempeño con fines de ingreso, promoción o permanencia realizados por el SPD; respecto a la dimensión formación continua se encontraron 24 documentos (24.7\%); y de instituciones y procesos de las políticas docentes, 15 archivos (15.4\%).

2 https://drive.google.com/file/d/1azZl3VbTYWygQTpebwd6k4LalCeFrup7/view 
Tabla 6. Distribución de los textos por año de publicación y dimensión

\begin{tabular}{lllll}
\hline & \multicolumn{2}{l}{ Dimensiones de las políticas docentes } & \\
\cline { 2 - 5 } Año & Carrera docente & Formación continua & $\begin{array}{l}\text { Instituciones y proce- } \\
\text { sos de las políticas do- } \\
\text { centes }\end{array}$ & Total \\
\hline 2013 & 3 & 0 & 1 & 4 \\
\hline 2014 & 0 & 1 & 1 & 2 \\
\hline 2015 & 1 & 2 & 7 & 10 \\
\hline 2016 & 6 & 2 & 0 & 8 \\
\hline 2017 & 17 & 7 & 1 & 25 \\
\hline 2018 & 18 & 3 & 2 & 23 \\
\hline 2019 & 12 & 8 & 2 & 22 \\
\hline 2020 & 1 & 1 & 1 & 97 \\
\hline Total & 58 & 24 & 15 & 3 \\
\hline
\end{tabular}

La mayor producción de textos se dio durante los últimos dos años del sexenio y el primero del siguiente. De hecho, la mayoría de las investigaciones realizadas por el INEE se publicaron en 2018.

Dimensiones de las políticas docentes. En la LGSPD (DOF, 2013c), la evaluación del desempeño docente y la formación continua del personal educativo se consideraron procesos transversales del SPD, en tanto que ambos están presentes a lo largo del ordenamiento para cada uno de los cuatro procesos del servicio civil de carrera para el profesorado mexicano de educación básica (Cordero et al., 2017). A partir de este planteamiento, las dimensiones carrera docente y formación continua se organizaron con base en las etapas de la carrera docente que corresponden a los procesos del SPD: a) ingreso e iniciación, b) promoción y reconocimiento, y c) permanencia. Además, se integró una categoría para incluir los documentos que se referían a $d$ ) los procesos del SPD en general.

Las etapas de ingreso e iniciación se relacionan con el proceso para acceder de manera formal al SPD y los primeros dos años de servicio (DOF, 2013c), en los que ocurre el periodo de inserción a la profesión docente (Vaillant, Marcelo, 2015). La promoción se refiere al "acceso a una categoría o nivel docente superior al que se tiene" (DOF, 2013c: 4), mientras que el reconocimiento se refiere"a las distinciones, apoyos y opciones de desarrollo profesional que se otorgan al personal que destaque en el desempeño de sus funciones" (DOF, 2013c: 4). En ambos casos, esto pudiera implicar cambio de funciones, ascensos o un mejor nivel de ingresos. La permanencia se entiende como la acción para evaluar el desempeño de los docentes y personal en funciones de dirección, asesoría técnica pedagógica o de supervisión, a fin de garantizar su idoneidad en el servicio educativo (DOF, 2013c). Finalmente, la subdimensión "Ios procesos del SPD en general" agrupa los textos que se referían a más de una de las etapas de la carrera docente. 
En el caso de la producción académica con respecto a la dimensión de estudios de instituciones y procesos de las políticas docentes, se subdividió en los ámbitos de análisis que se identificaron en los textos: $a$ ) instituciones, $b$ ) leyes y documentos oficiales y $c$ ) percepciones y reflexiones del profesorado. La tabla 7 muestra los textos identificados en cada dimensión y subdimensión.

Tabla 7. Concentrado de los textos por dimensión y subdimensión

\begin{tabular}{|c|c|c|c|c|}
\hline Dimensión & Subdimensión & Textos & $N$ & $\%$ \\
\hline \multirow{4}{*}{ Carrera docente } & $\begin{array}{l}\text { Ingreso e inicia- } \\
\text { ción }\end{array}$ & $\begin{array}{l}{\left[\mathrm{P}_{40}\right]\left[\mathrm{A}_{1} 6\right]\left[\mathrm{A}_{14}\right][\mathrm{E} 08][\mathrm{Eog}]\left[\mathrm{E}_{20}\right]} \\
{\left[\mathrm{E}_{21}\right]}\end{array}$ & 7 & 7.2 \\
\hline & Permanencia & 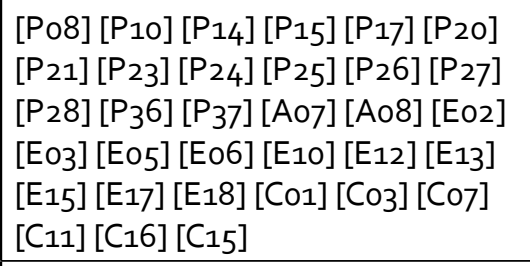 & 33 & 34.0 \\
\hline & $\begin{array}{l}\text { Promoción y } \\
\text { reconocimiento }\end{array}$ & {$\left[E_{22}\right]\left[E_{16}\right]\left[C_{12}\right]$} & 3 & 3.1 \\
\hline & $\begin{array}{l}\text { Procesos de eva- } \\
\text { luación del SPD } \\
\text { en general }\end{array}$ & $\begin{array}{l}{\left[\mathrm{P}_{40}\right]\left[\mathrm{A}_{01}\right][\mathrm{A} 09]\left[\mathrm{A}_{10}\right]\left[\mathrm{A}_{11}\right]\left[\mathrm{A}_{12}\right]} \\
{\left[\mathrm{A}_{13}\right]\left[\mathrm{A}_{15}\right]\left[\mathrm{A}_{17}\right]\left[\mathrm{A}_{20}\right]\left[\mathrm{E}_{4}\right]\left[\mathrm{E}_{14}\right]} \\
{[\mathrm{E} 19][\mathrm{Co}]\left[\mathrm{C}_{14}\right]}\end{array}$ & 15 & $15 \cdot 5$ \\
\hline \multirow{4}{*}{$\begin{array}{l}\text { Formación con- } \\
\text { tinua }\end{array}$} & $\begin{array}{l}\text { Ingreso e inicia- } \\
\text { ción }\end{array}$ & $\begin{array}{l}{\left[\mathrm{P}_{05}\right]\left[\mathrm{P}_{30}\right]\left[\mathrm{P}_{31}\right]\left[\mathrm{P}_{32}\right]\left[\mathrm{P}_{39}\right]\left[\mathrm{P}_{41}\right]} \\
{[\mathrm{Eo1}]}\end{array}$ & 7 & 7.2 \\
\hline & Permanencia & {$\left[\mathrm{P}_{11}\right]\left[\mathrm{P}_{13}\right]\left[\mathrm{P}_{34}\right]\left[\mathrm{Ao3}_{0}\right]$} & 4 & 4.1 \\
\hline & $\begin{array}{l}\text { Promoción y } \\
\text { reconocimiento }\end{array}$ & {$\left[\mathrm{P}_{12}\right]\left[\mathrm{P}_{18}\right]\left[\mathrm{P}_{19}\right]\left[\mathrm{P}_{22}\right]\left[\mathrm{P}_{33}\right]\left[\mathrm{A}_{06}\right]$} & 6 & 6.2 \\
\hline & $\begin{array}{l}\text { Procesos de } \\
\text { formación en } \\
\text { general }\end{array}$ & $\begin{array}{l}{\left[\mathrm{P}_{35}\right]\left[\mathrm{Ao5}_{0}\right][\mathrm{E} 07]\left[\mathrm{E}_{11}\right][\mathrm{Co}][\mathrm{Co}]} \\
{\left[\mathrm{C}_{17}\right]}\end{array}$ & 7 & 7.2 \\
\hline \multirow{3}{*}{$\begin{array}{l}\text { Instituciones y } \\
\text { procesos de las } \\
\text { políticas docen- } \\
\text { tes }\end{array}$} & Instituciones & [Po1] [Po6] [Co2] [Cog] [C10] & 5 & 5.2 \\
\hline & $\begin{array}{l}\text { Leyes y docu- } \\
\text { mentos oficiales }\end{array}$ & {$\left[\mathrm{Po}_{3}\right]\left[\mathrm{Po}_{4}\right]\left[\mathrm{Ao}_{4}\right]\left[\mathrm{A}_{18} 8\right]\left[\mathrm{CO}_{5}\right]\left[\mathrm{C}_{13}\right]$} & 6 & 6.2 \\
\hline & $\begin{array}{l}\text { Percepciones y } \\
\text { reflexiones del } \\
\text { profesorado }\end{array}$ & {$[\mathrm{Po} 7]\left[\mathrm{P}_{16} 6[\mathrm{A02}]\left[\mathrm{A}_{19}\right]\right.$} & 4 & 4.1 \\
\hline Total & & & 97 & 100.0 \\
\hline
\end{tabular}

En cuanto al análisis de las dimensiones, carrera docente fue el tema más estudiado y, dentro de éste, la evaluación del desempeño con fines de permanencia, seguida de los procesos de evaluación en general. En la dimensión formación continua, las subdimensiones más estudiadas fueron ingreso e iniciación y procesos de formación en general. En contraste, la formación 
continua en los procesos de permanencia fue el tema menos analizado. En instituciones y procesos de las políticas docentes los estudios se desarrollaron en el siguiente orden: leyes y documentos oficiales, instituciones, y percepciones y reflexiones, no hubo una diferencia sobresaliente entre el número de textos en cada subdimensión.

Finalmente, si se revisa la tabla de acuerdo con las subdimensiones, al comparar el número de textos publicados con respecto a los procesos transversales del SPD, es decir evaluación y formación, se encontraron 14 textos con referencia al ingreso e iniciación; de estos, siete centrados en la evaluación y siete en la formación. En cuanto a la permanencia, se identificó que el proceso más estudiado fue la evaluación, pues 33 documentos tuvieron como objeto de estudio ese proceso, mientras que sólo cuatro de los textos se centraron en la formación continua asociada con la permanencia. Respecto a la promoción y reconocimiento, sólo tres de los textos se refirieron a la evaluación en estos procesos y seis a la formación continua. De los 60 trabajos relativos a carrera docente y formación continua, 43 refieren específicamente a evaluación del desempeño con fines de ingreso, permanencia y promoción, y sólo 17 atienden a la formación asociada a estos tres procesos.

\section{Conclusiones}

Un mapeo sistemático permite describir el comportamiento métrico de una muestra de literatura seleccionada a partir de ciertos criterios. Ofrece un mapa accesible para identificar en el cúmulo de la producción una guía que oriente y facilite su búsqueda, localización y acceso al conocimiento científico, haciendo frente a la complejidad de este proceso (Wagner et al., 1999), por lo que la contribución de los mapeos a la literatura es la organización de la producción y no las abstracciones teóricas o su discusión.

Este estudio es un mapeo sistemático de la producción académica en el periodo 2013-2020 respecto a las políticas docentes características del ejercicio de la Reforma Educativa de 2013 para el profesorado en servicio. Responde a la interrogante de cómo la investigación educativa abordó las políticas docentes que se definieron constitucional y legalmente el sexenio pasado.

Del conjunto de políticas docentes, se mapearon tres dimensiones: carrera docente, formación continua y estudios de instituciones y procesos de las políticas docentes. Se encontraron 97 documentos, 39.2\% fueron ponencias presentadas en el Congreso Mexicano de Investigación Educativa, lo cual da evidencia de una producción de conocimiento y difusión generalizada gracias al marco de este importante congreso nacional. 17.5\% fueron capítulos de libros, producto que es menos accesible para autores y lectores por los costos asociados.

El INEE, como organismo autónomo, realizó una diversidad de estudios sobre políticas docentes, un cuarto de la producción fue generada por este instituto. Principalmente se documentaron las distintas evaluaciones del SPD, aunque también se identificaron documentos referidos a la formación continua de los profesores de educación básica. Si bien el instituto fue derogado 
el 15 de mayo de 2019, aún es posible acceder a la diversidad de sus publicaciones que bien valdría la pena recuperar para trazar líneas de investigación.

La producción académica se concentró de 2017 a 2019, con 72\% de la producción, lo cual se entiende por el hecho de que para esas fechas ya se habían aplicado todos los procesos definidos por la LGSPD y por la polémica política que se desató a finales del sexenio en torno a su cancelación como promesa de campaña presidencial.

Se puede concluir que el interés central de la producción académica que refiere a las políticas docentes durante la Reforma Educativa de 2013 fue la carrera docente. Se encontró un mayor número de trabajos asociados a esta dimensión (59.7\%). La abundancia en los estudios sobre carrera docente que comprenden la temática de la evaluación del desempeño docente con fines de ingreso, promoción y permanencia, es acorde con la centralidad que estos temas adquirieron en la política educativa nacional (Cuevas, Rangel, 2019) y está asociada al interés originado por el alto impacto que dicho proceso tenía en la vida laboral del profesorado, en particular, la evaluación del desempeño con fines de permanencia.

La formación continua recibió menor atención por parte de la comunidad académica; solamente una cuarta parte de la producción identificada abordó esta temática. Se coincide con Vezub (2019: 4), quien planteó que "todavía resulta insuficiente la información disponible y consolidada sobre las acciones que los diferentes países realizan con el propósito de elevar el desarrollo profesional de los educadores en servicio". El programa más estudiado fue el Programa de Tutoría y Acompañamiento, por lo que se puede concluir que hubo un particular interés por las políticas de inserción al servicio, en virtud de que ésta era una de las novedades del SPD. Esta etapa ha sido ampliamente estudiada en otros países (Vaillant, Marcelo, 2015), pero en México no había sido reconocida, hasta su incorporación en la LGSPD como una etapa específica de formación. La formación asociada a los procesos de permanencia en el servicio fue una de las subdimensiones menos analizadas.

A pesar del paralelismo entre evaluación y formación indicado por la LGSPD, y del hecho de que esta ley definió un modelo de formación continua, la formación en el servicio no fue cabalmente estudiada ni atendida en el sexenio (Cordero, 2018). Se concluye que es necesario brindar mayor atención al análisis de las políticas de formación continua en su conjunto, a fin de contribuir a darles centralidad, no sólo en el discurso político sino también en su puesta en operación y evaluación (Vezub, 2019).

En tercer término, se ubicaron los estudios de las instituciones y procesos de diseño e implantación de las políticas docentes. Su producción fue escasa (15.45\%) en comparación los otros dos temas.

En cuanto a las ausencias en la investigación, se reconoce la carencia de investigación de la experiencia particular de la implementación del SPD en las entidades federativas. Hacen falta más estudios que aporten conocimientos sobre contextos concretos en la diversidad del país 
y las decisiones que tomaron las autoridades educativas estatales para atender las demandas de la LGSPD. Además, se identificó que se carece de estudios que analicen de forma integral las políticas docentes derivadas de la Reforma Educativa de 2013.

El tema de las políticas docentes es polémico, lo fue en el sexenio pasado (Ornelas, 2018) y lo sigue siendo este sexenio, ante las críticas que se hacen a la operación de los procesos de carrera docente que se han instrumentado (Martínez Dunstan, 2021). Los resultados de este trabajo son un instrumento de apoyo para los interesados en entender las lecciones del pasado, comprender el origen de las decisiones del presente y buscar mejores opciones de futuro en el tema de políticas docentes (Del Castillo, 2019). Así, este mapeo proporciona información sistematizada para que el lector trace rutas propias de exploración que deriven en una revisión sistemática de la literatura.

\section{Referencias}

Ávalos, B. (2007). Formación docente continua y factores asociados a la política educativa en América Latina y el Caribe. Informe preparado para el Diálogo Regional de Política Banco Interamericano de Desarrollo.

Birgin, A. (2012). Más allá de la capacitación. Debates acerca de la formación de los docentes en ejercicio. Buenos Aires: Paidós.

Bruns, B.; J. Luque (2015). Profesores excelentes. Cómo mejorar el aprendizaje en América Latina y el Caribe. Estados Unidos: Banco Mundial. https://openknowledge.worldbank.org/handle/10986/20488

Celdrán-Bernabeu, M.; J. Mazón; J. Ivars-Baidal; J.Vera-Rebollo (2018). Smart Tourism. Un estudio de mapeo sistemático. Cuadernos de Turismo, (41), 107-138. https://doi.org/10.6018/turismo.41.326971

Celaya, l.; M. Ramírez-Montoya; C. Naval-Durán; E. Arbués (2020). Usos del podcast para fines educativos. Mapeo sistemático de la literatura en WoS y Scopus (2014-2019). Revista Latina de Comunicación Social, 77, 179-201. https://doi.org/10.4185/RLCS-2020-1454

Cordero, G.; J. Jiménez; C. Navarro; M. Vázquez (2017). Diagnóstico de la política pública de formación y desarrollo profesional del personal educativo de educación básica de la Reforma Educativa. México: INEE. https://www.inee.edu.mx/wp-content/uploads/2019/01/P1F214.pdf

Cordero, G. (2018). Desafíos de la formación docente. En Guevara-Niebla, G.; E. Backhoff (coords.). La Reforma Educativa. Avances y desafíos. México: Fondo de Cultura Económica, 200-209.

Cuevas, Y.; K. Rangel (2019). Análisis de la carrera docente en la educación primaria en México: Entre el credencialismo y la meritocracia. Archivos Analíticos de Políticas Educativas, 27(44). http://dx.doi.org/10.14507/epaa.27.4323 
Cuevas, Y.; C. Inclán (2021). Políticas docentes en América Latina: Diseño, implementación y experiencias. Revista Mexicana de Investigación Educativa, 26(89), 351-367. http://www.comie. org.mx/v5/sitio/wp-content/uploads/2021/04/RMIE 89.pdf

Cuenca, R. (2015). Las carreras docentes en América Latina. La acción meritocrática para el desarroIlo profesional. Estrategia regional sobre docentes. Chile: Orealc/Unesco https://teachertaskforce.org/sites/default/files/2020-07/las carreras docentes sp.pdf

Del Castillo, G. (2019). Reflexión sobre la iniciativa de reforma del artículo $3^{\circ}$ constitucional [Conferencia]. Palacio Legislativo: Comisiones Unidas de Educación y Puntos Constitucionales. México. http://www5.diputados.gob.mx/index.php/esl/content/download/137730/688456/ file/Gloria\%20del\%20Castillo\%20Alem\%C3\%A1n.pdf

Diario Oficial de la Federación (2013a). Decreto por el que se reforman los artículos 3o., en sus fracciones III, VII y VIII; y 73, fracción XXV, y se adiciona un párrafo tercero, un inciso d) al párrafo segundo de la fracción II y una fracción IX al artículo 3o. de la Constitución Política de los Estados Unidos Mexicanos. http://www.dof.gob.mx/nota detalle.php?codigo=528891 9\&fecha $=26 / 02 / 2013$

Diario Oficial de la Federación (2013b). Ley General de Educación. http://dof.gob.mx/nota detalle.php?codigo $=5313841 \&$ fecha $=11 / 09 / 2013 \#: \sim:$ tex$\mathrm{t}=\% 2 \mathrm{D} \% 20$ Todo\%20individuo\%20tiene\%20derecho\%20a,establezcan\%20las\%20disposiciones\%20generales\%20aplicables

Diario Oficial de la Federación (2013c). Ley General del Servicio Profesional Docente. https://www. sep.gob.mx/work/models/sep1/Resource/558c2c24-0b12-4676-ad90-8ab78086b184/ley general servicio profesional docente.pdf

Diario Oficial de la Federación (2013d). Ley del Instituto Nacional para la Evaluación de la Educación. https://www.sep.gob.mx/work/models/sep1/Resource/558c2c24-0b12-4676-ad908ab78086b184/ley instituto nacional evaluacion educativa.pdf

Ducoing, P. (coord.). (2019). Programas y políticas de evaluación docente en educación básica (1993-2017). México: Instituto de Investigaciones sobre la Universidad y la Educación IISUEUNAM.

Elacqua, G.; D. Hincapie; E. Vegas; M. Alfonso (2018). Profesión: profesor en América Latina. ¿Por qué se perdió el prestigio docente y cómo recuperarlo? Estados Unidos: Banco Mundial. http:// dx.doi.org/10.18235/0001172

Keele, S. (2007). Guidelines for Performing Systematic Literature Reviews in Software Engineering (vol. 5). Technical Report, ver 2.3 EBSE Technical Report. EBSE.

Martínez, S. (2021). Los derechos laborales y los derechos profesionales del magisterio. Educación Futura. http://www.educacionfutura.org/los-derechos-laborales-y-los-derechos-profesionales-del-magisterio/ 
Mexicanos Primero (2016). Informe anual 2016. México. https://s3-us-west-2.amazonaws.com/ static-mexicanosprimero.org/informes/informeanual mp 2016.pdf

Monroy, M.; J. Arciniegas; J. Rodríguez (2016). Recuperación de arquitecturas de software: un mapeo sistemático de la literatura. Información Tecnológica, 27(5): 201-220. http://dx.doi. org/10.4067/S0718-07642016000500022

Murillo, J. (2007). Evaluación del desempeño y carrera profesional docente. Un estudio comparado entre 50 países de América y Europa. Chile: OREALC-UNESCO. https://opech.cl/ doc/ evaluacion desempeno carrera profesional docente unesco.pdf

Navarro, C.; M. Ramírez (2018). Mapeo sistemático de la literatura sobre evaluación docente (2013-2017). Educação e Pesquisa, (44). https://www.scielo.br/scielo.php?script=sci abstract\&pid=S1517-97022018000100498\&lng=pt\&nrm=is\&tlng=es

Oficina Regional de Educación para América Latina y el Caribe-Organización de las Naciones Unidas para la Educación, la Ciencia y la Tecnología (2013). Antecedentes y criterios para la elaboración de políticas docentes en América Latina y el Caribe. Chile. https://unesdoc. unesco.org/ark:/48223/pf0000223249

Ornelas, C. (2018). La contienda por la educación. México: Fondo de Cultura Económica.

Paoli, F. (2013). Elementos relevantes de la reforma educativa. En Ramírez, R. (coord.). La reforma constitucional en materia educativa: alcances y desafíos. México: Instituto Belisario Domínguez, Senado de la República, 169-177. http://bibliodigitalibd.senado.gob.mx/handle/123456789/2937

Petersen, K.; R. Feldt; S. Mujtaba; M. Mattsson (2008). Systematic Mapping Studies in Software Engineering. EASE'08 Proceedings of the 12th International Conference on Evaluation and Assessment in Software Engineering (EASE). British Computer Society Swinton, 68-77. https:// dl.acm.org/doi/10.5555/2227115.2227123

Rodríguez, R. (2016). Reformar la reforma, evaluar la evaluación. En Foro Hacia una Verdadera Reforma Educativa. México. http://www.ses.unam.mx/integrantes/uploadfile/rrodriguez/ Rodriguez2016 HaciaUnaVerdaderaReformaEducativa.pdf

Sinoara, R.; J. Antunes; S. Rezende (2017). Text Mining and Semantics: A Systematic Mapping Study. Journal of the Brazilian Computer Society, 23(9), 7-22. https://doi.org/10.1186/s13173017-0058-7

Vaillant, D.; C. Marcelo (2015). El ABC y D de la formación docente. España: Nárcea.

Vezub, L. (2019). Las políticas de formación docente continua en América Latina. Mapeo exploratorio en 13 países. IIPE-UNESCO. https://www.buenosaires.iiep.unesco.org/sites/default/files/ archivos/An\%C3\%A1lisis\%20comparativo.\%20Lea\%20Vezub.pdf

Vezub, L. (2021). Investigar la formación continua y fortalecer el campo de la formación docente. En Cordero, G.; J. García-Poyato; K. Rivera (coords.). Evaluación de la formación continua de docentes: investigaciones desde la Escuela Normal en Baja California. México: Juan Pablos Editor, 29-34. 
Villar, A., S. Matalonga (2013). Definiciones y tendencia de deuda técnica: Un mapeo sistemático de Ia literatura. Uruguay: ClbSE, Universidad ORT.

Wagner, P.; C. Weiss; B. Wittrock; H. Wollman (1999). Ciencias sociales y Estados modernos: experiencias nacionales e incidencias teóricas. México: Fondo de Cultura Económica. 